\title{
Modeling ionization and recombination from low energy nuclear recoils in liquid argon
}

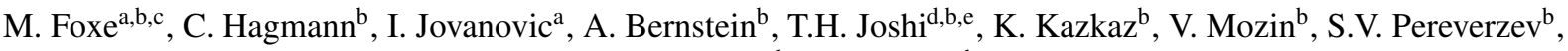 \\ S. Sangiorgio ${ }^{\mathrm{b}}$, P. Sorensen ${ }^{\mathrm{b}}$ \\ ${ }^{a}$ Department of Mechanical and Nuclear Engineering, The Pennsylvania State University, University Park, PA 16802, USA \\ ${ }^{b}$ Lawrence Livermore National Laboratory, Livermore, CA 94550, USA \\ ${ }^{c}$ Pacific Northwest National Laboratory, Richland, WA 99352, USA \\ ${ }^{d}$ Department of Nuclear Engineering, University of California, Berkeley, CA 94720, USA \\ ${ }^{e}$ Present address: Lawrence Berkeley National Laboratory, 1 Cyclotron Road, Berkeley, CA 94720, USA
}

\begin{abstract}
Coherent elastic neutrino-nucleus scattering (CENNS) is an as-yet undetected, flavor-independent neutrino interaction predicted by the Standard Model. Detection of CENNS could offer benefits for detection of supernova and solar neutrinos in astrophysics, or for detection of antineutrinos for nuclear reactor monitoring and nuclear nonproliferation. One challenge with detecting CENNS is the low energy deposition associated with a typical CENNS nuclear recoil. In addition, nuclear recoils result in lower ionization yields than those produced by electron recoils of the same energy. While a measurement of the nuclear recoil ionization yield in liquid argon in the keV energy range has been recently reported, a corresponding model for low-energy ionization yield in liquid argon does not exist. For this reason, a Monte Carlo simulation has been developed to predict the ionization yield at sub-10 keV energies. The model consists of two distinct components: (1) simulation of the atomic collision cascade with production of ionization, and (2) the thermalization and drift of ionization electrons in an applied electric field including local recombination. As an application of our results we report updated estimates of detectable ionization in liquid argon from CENNS at a nuclear reactor.
\end{abstract}

Keywords: liquid argon, ionization yield, neutrino, dark matter

\section{Introduction}

Dual-phase noble element detectors are extensively used in the search for dark matter [1, 2, 3, 4, 5, 6, 7, but they are also of great interest in the quest to detect coherent elastic neutrino-nucleus scatter (CENNS) [8, 9]. The reasons for their extensive use include the scalability, high sensitivity [10, 11], favorable electron drift properties [12, 13], and relatively low cost for a dualphase xenon (Xe) or argon (Ar) detector. It is important to understand that we are nearing the absolute limits of sensitivity of those detectors, i.e. detection of very few electron-ion pairs and photons produced by primary interaction. In addition, recoiling nuclei (or ions) transfer a large fraction of their energy to heat, in contrast to ionization by electrons, where most of the energy is expended on ionization and photon production. Therefore, an understanding of physical processes involved in

Email address: Michael.Foxe@pnnl.gov (M. Foxe) detector operation at low energies is becoming increasingly important.

A variety of models have been used in the past in an effort to predict the ionization yield (defined as the number of electrons produced per energy deposited) at low energies $(\mathrm{keV})$ in liquid noble element detectors. The Lindhard theory [14, 15] is successful in predicting the ionization yield at higher energies $(\mathrm{MeV})$, but requires an independent knowledge of the ratio of energies expended to ionization and scintillation at lower energies [16], and an additional semi-empirical proportionality factor is needed to reconcile it with the measurements. Other semi-empirical models have been constructed, aimed at predicting the nuclear quench factors and scintillation yields in a variety of scintillator materials including noble liquids [17, 18, 19]. While capable of producing good fits to the experimental detector response data, those models ignore specific reconstruction of the spatial structure of ionization tracks at low ener- 
gies.

An atomistic Monte Carlo model has been previously developed to predict the nuclear quench factor in liquid argon (LAr) [8] at energies relevant for CENNS experiments conducted using antineutrinos produced by nuclear reactors. In this current work, we refine the atomistic Monte Carlo model [8] by including $\mathrm{Ar}^{+}$cross sections and the effects of local recombination in an applied drift field [20]. Though we do not estimate final uncertainties on quantitative predictions, our modeling accurately describes qualitative features of the ionization tracks at low energies which need to be taken into account in existing empirical models.

In addition to the expected ionization yields for nuclear recoil energies up to $\sim 10 \mathrm{keVr}$, we present a revised prediction for the CENNS ionization yields produced from reactor antineutrinos.

\section{Primary Ionization Yield Using Atomic Collision Monte Carlo Simulation}

The algorithm used in the TRansport of Ions in Matter (TRIM) [21]-based binary collision Monte Carlo model has been described in previous work [8]. It handles the elastic Coulomb deflections of the incident fast $\mathrm{Ar}$ atom in the medium during slowing down. For the energy range considered here, most of the energy in the slowing down process is transferred to other atoms in elastic Coulomb collisions, thus dominating the stopping power $-d E / d x$. The remainder of the energy is transferred through inelastic collisions.

In this work we have included the ionization and excitation of a neutral $\mathrm{Ar}$ atom by another $\mathrm{Ar}$ atom or $\mathrm{Ar}^{+}$ ion and charge exchange between a fast $\mathrm{Ar}^{+}$ion and a neutral background Ar atom. Figures 1 and 2 show the inelastic cross sections compiled by Phelps [22] for incident $\mathrm{Ar}$ and $\mathrm{Ar}^{+}$projectiles, respectively. All ionization and excitation cross sections are small compared to the geometric cross section $\sigma_{g e o}=n^{-2 / 3}$ for elastic Coulomb deflections, where $n$ is the Ar number density [8, 21].

The excitation producing 811 and $795 \mathrm{~nm}$ photons is ignored for this simulation. The $811 \mathrm{~nm}$ and $795 \mathrm{~nm}$ cross sections are $10 \%$ and $2.5 \%$ of the ionization/UV cross section, respectively [22]. For a $10 \mathrm{keV}$ recoil, it is estimated that $<100 \mathrm{eV}$ would be lost to the production of 811 and $795 \mathrm{~nm}$ photons.

We take the probability for ionization occurring in 112 a Coulomb collision as $p_{i o n}=\sigma_{i o n} / \sigma_{g e o}$, where $\sigma_{i o n} 113$ is the ionization cross section; an analogous expres- 114 sion is used for excitation. In every Coulomb colli- 115 sion the inelastic channels are sampled based on those 116 probabilities and are uncorrelated with the scattering angle. One of the outgoing particles is randomly chosen to be $\mathrm{Ar}^{+}$for ionization events and similarly $\mathrm{Ar}^{*}$ for excitations. Due to the large charge transfer cross section, $\sigma_{C T}$, few $\mathrm{Ar}^{+}$atoms survive to the next collisions event, with a survival probability estimated as $p_{\text {sur }}=\exp \left(-\sigma_{C T} / \sigma_{g e o}\right)$. The leading particle plus all secondaries with kinetic energies larger than the excitation or ionization threshold $(\sim 25 \mathrm{eV}$ in the laboratory frame) are tracked as a part of the collision cascade.

Other approximations made include: (1) the inelastic cross sections of a fast $\mathrm{Ar}^{*}$ atom are taken to be the same as an Ar atom in the ground state due to the lack of data, and (2) the target atom in a collision is assumed to be a neutral Ar in the ground state. The additional energy lost in an inelastic collision is taken from the more energetic outgoing particle to eliminate the possibility of a particle acquiring negative energy.

Unfortunately, the experimental uncertainties of the cross sections used in this simulation are not known. Also, while the TRIM algorithm is often used for problems similar to the one considered here, we are unaware of a solid treatment in the literature of systematic uncertainties arising from neglecting three-body collisions in modeling ionization dynamics in liquid.

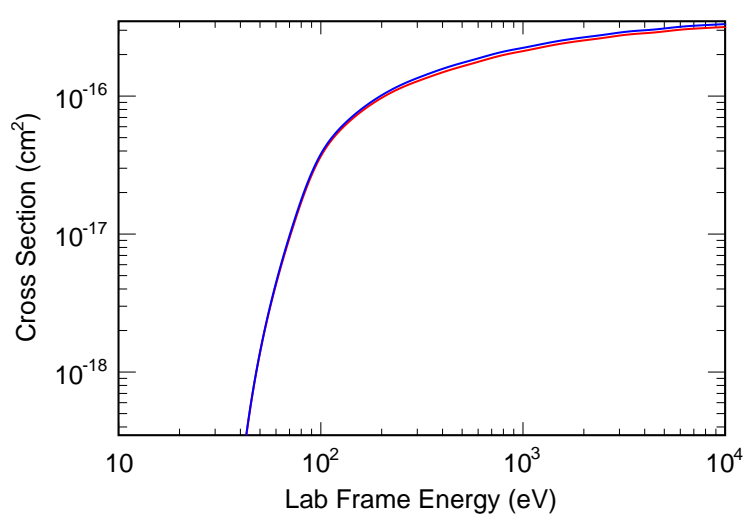

Figure 1: In a given neutral-neutral $(\mathrm{Ar}+\mathrm{Ar})$ collision, elastic scattering, ionization, or excitation can occur. Shown are the cross sections for ionization (red), $\mathrm{Ar}+\mathrm{Ar} \rightarrow \mathrm{Ar}+\mathrm{Ar}^{+}+e^{-}$, and excitation (blue), $\mathrm{Ar}+\mathrm{Ar} \rightarrow \mathrm{Ar}+\mathrm{Ar}^{*}$, based on tabulated values in Reference [22]. The cross sections are nearly equal, with excitation cross section being slightly higher than ionization cross section, which results in similar rates of primary excitation and ionization.

The ionization electron spectrum is not well known and evidence exists that it depends on the collision energy [23]. For our studies of electron drift and recombination, we chose two discrete electron energies, $E_{\text {elec }}=3$ and $10 \mathrm{eV}$, based on the electron spectrum for 


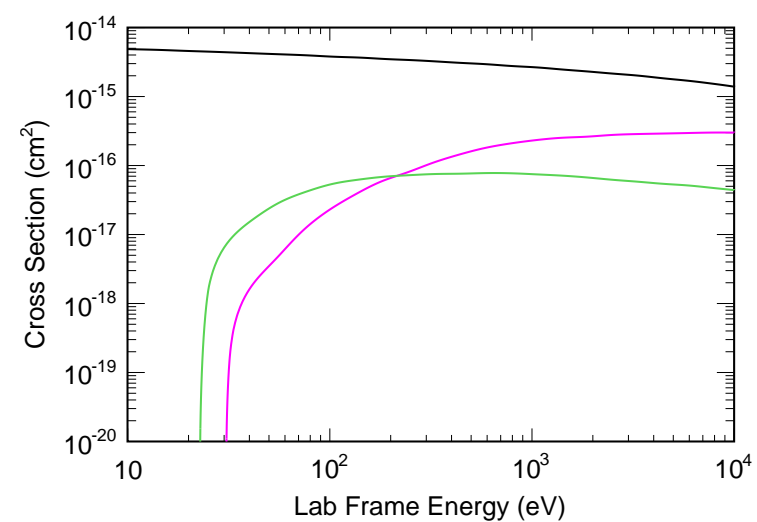

Figure 2: Cross sections for charge transfer (black), $\mathrm{Ar}^{+}+\mathrm{Ar}$ $\rightarrow \mathrm{Ar}+\mathrm{Ar}^{+}$, ionization (magenta), $\mathrm{Ar}^{+}+\mathrm{Ar} \rightarrow \mathrm{Ar}^{+}+\mathrm{Ar}^{+}+\mathrm{e}^{-}$, and excitation (green), $\mathrm{Ar}^{+}+\mathrm{Ar} \rightarrow \mathrm{Ar}^{+}+\mathrm{Ar}^{*}$, based on tabulated values in Reference [22].

Table 1: Material specific properties of LAr used in the atomic collision model.

\begin{tabular}{|c|c|c|c|}
\hline Property & Variable & Value & Units \\
\hline Atom Density & $n$ & $2.11 \times 10^{22}$ & $\mathrm{~cm}^{-3}$ \\
Coll. Path Length & $L_{g e o}$ & 3.62 & $\AA$ \\
Geo. Cross Section & $\sigma_{g e o}$ & $1.31 \times 10^{-15}$ & $\mathrm{~cm}^{2}$ \\
Ionization Potential & $I_{p}$ & 14.3 & $\mathrm{eV}$ \\
Excitation Energy & $E_{\text {exc }}$ & 12 & $\mathrm{eV}$ \\
Ion. Elec. Energy & $E_{\text {elec }}$ & 3,10 & $\mathrm{eV}$ \\
\hline
\end{tabular}

Ar-Ar collisions in Ref [23]. Material-specific input parameters taken into account in the simulation are listed in Table 1 and a summary of all treated interactions is provided in Table 2

The range of $\mathrm{Ar}$ atoms with $\mathrm{keV}$ energies in $\mathrm{LAr},{ }_{138}$ shown in Figure 3 , is short compared to the range of electrons of the same energy. The Ar ion recoil is simulated in this work, while electron recoils have been previously simulated in [20].

The primary ionization yields were obtained by averaging 10000 collision histories for each incident energy. The positions of the Ar ionization sites were recorded for the recombination stage of the simulation. Figure 4 shows the average number of produced ionization elec- ${ }_{147}$ trons as well as their dispersion which affects energy ${ }_{148}$ resolution. The ionization yield is only slightly $(\sim 2 \%){ }_{149}$ larger than the ionization yield obtained perviously $[8]_{150}$ by tracking only neutral Ar atoms.

The primary scintillation and ionization yields in our ${ }_{152}$ current model are nearly identical, because ionization ${ }_{153}$
Table 2: A summary of the possible interactions between neutral and charged $\mathrm{Ar}$ atoms as they slow down. Here, $I_{p}$ is the ionization potential, $E_{\text {elec }}$ is the ejected electron energy, and $E_{\text {exc }}$ is the atomic excitation energy. 'COM' is center of mass.

\begin{tabular}{|c|c|c|c|}
\hline Particle & Target & Collision Type & COM $E$ Loss \\
\hline $\mathrm{Ar}$ & $\mathrm{Ar}$ & Elastic & 0 \\
$\mathrm{Ar}$ & $\mathrm{Ar}$ & Ionization & $E_{\text {elec }}+I_{p}$ \\
$\mathrm{Ar}$ & $\mathrm{Ar}$ & Excitation & $E_{\text {exc }}$ \\
$\mathrm{Ar}^{+}$ & $\mathrm{Ar}$ & Charge Transfer & 0 \\
$\mathrm{Ar}^{+}$ & $\mathrm{Ar}$ & Ionization & $E_{\text {elec }}+I_{p}$ \\
$\mathrm{Ar}^{+}$ & $\mathrm{Ar}$ & Excitation & $E_{\text {exc }}$ \\
\hline
\end{tabular}

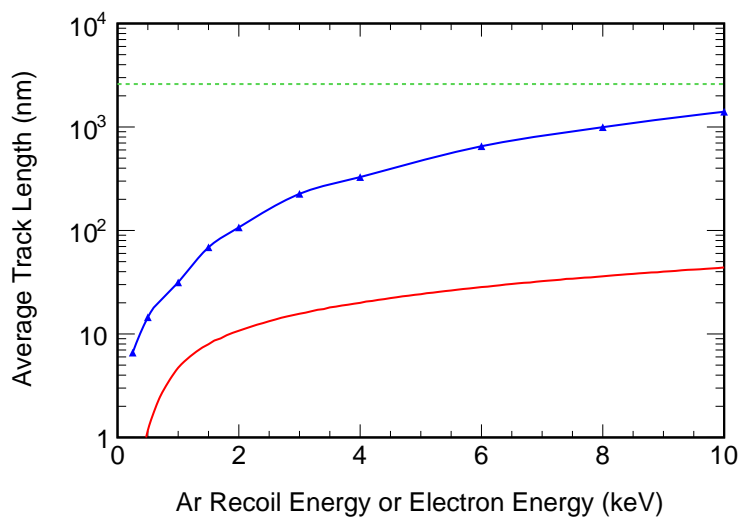

Figure 3: The range of the Ar atoms in LAr (red) is on the order of nm, which is much shorter than the range of electrons of the same energy (blue) [20]. Both ranges come from the respective simulations. The ionization electron thermalization length of $2.6 \mu \mathrm{m}$ (green-dashed) is very weakly dependent on the secondary electron energy in the range of $1-5 \mathrm{eV}$ [24].

and excitation collisions cross sections for Ar-Ar collisions are very nearly the same (Figure 11). The difference between inelastic cross sections for $\mathrm{Ar}_{\text {and }} \mathrm{Ar}^{+}$ (Figures 1 and 2 ) becomes unimportant for the accuracy of the simulation due to the high probability of charge transfer. On the other hand, Figure 3 indicates that distances between exited Ar atoms of the compact 'positive core' became small and comparable to interatomic distances. At these conditions one should expect effective pair-recombination of excited states due to Penning ionization, without requiring direct collision of exited atoms, because electron tunneling is involved in the Penning process: $\mathrm{Ar}^{*}+\mathrm{Ar}^{*} \rightarrow \mathrm{Ar}^{+}+\mathrm{e}^{-}+\mathrm{Ar}$. As a result, at low recoil energies, ionization yield should be somewhat higher at the expense of primary scintillation. Unfortunately, data on Penning ionization in LAr is not available, in contrast to liquid $\mathrm{He}$, where this pro- 


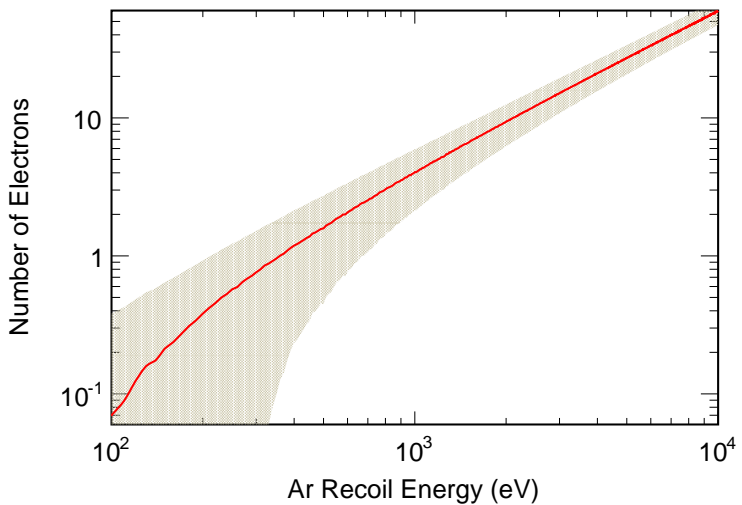

Figure 4: The predicted electron yield including the $\mathrm{Ar}^{+}$collision cross sections and $E_{\text {elec }}=3 \mathrm{eV}$ (red) and dispersion (brown error band) for the recoiling Ar atoms. For comparison, the electron yield without the $\mathrm{Ar}^{+}$collision cross sections [8] is approximately 1 electron fewer $(\sim 2 \%)$ at a recoil energy of $10 \mathrm{keV}$.

\section{cess has been extensively studied both theoretically [25} and experimentally [26].

\section{The Model of Electron Recombination and Elec- tron Separation Efficiency}

In addition to calculating how many electrons are created in a given recoil, the fraction of electrons which escape recombination also needs to be calculated. The simulation of the electron drift is based on the CohenLekner theory [27], as described by Wojcik [28]. The method for simulating electron drift and recombination for incident electron energies up to $10 \mathrm{keV}$ has been presented in [20]. The position of the secondary electrons is obtained from the atomistic Monte Carlo, and the subsequent transport is performed using the framework described in Ref [20].

Tallying the number of electrons which survive local recombination after thermalization and drift in the externally applied field yields the electron separation efficiency ('electron extraction efficiency' is usually used to describe the process of extraction of electrons from the liquid into the gas phase in dual-phase detectors). This calculation is performed for various electric fields and for two representative electron emission energies from the Ar atom: $3 \mathrm{eV}$ and $10 \mathrm{eV}$ [23], Figure 5] Little difference is seen in the charge separation efficiency for 206 different electron emission energies. This is because the 207 electron thermalization length is only weakly dependent 208 on initial electron energies for the large $(>1 \mathrm{eV})$ elec- 209 tron energies; calculations for the range of $1-5 \mathrm{eV}$ were 210 performed in Ref. [24]. Thus, we can disregard the 211

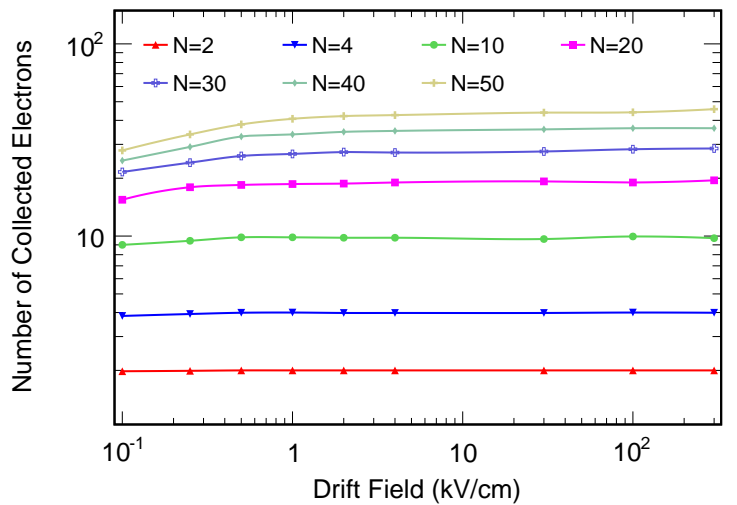

Figure 5: Calculated number of collected electrons as a function of drift field for variable number of initial ionizations for $E_{\text {elec }}=3 \mathrm{eV}$. The electron escape efficiency is near unity for all fields and $\mathrm{N}<\sim 10$. For larger N, the Coulomb attraction between electrons and the dense core of positive ions becomes important resulting in substantial recombination. Little difference is observed when an ionization electron emission energy of $10 \mathrm{eV}$ is used.

The dependence of the escape efficiency as a function
of drift field is very similar for Ar and electron recoils [20] for the same number of released electrons. The reason for this are the short track lengths of both projectiles compared to the thermalization distance of the ionization electrons, as shown in Figure 3 


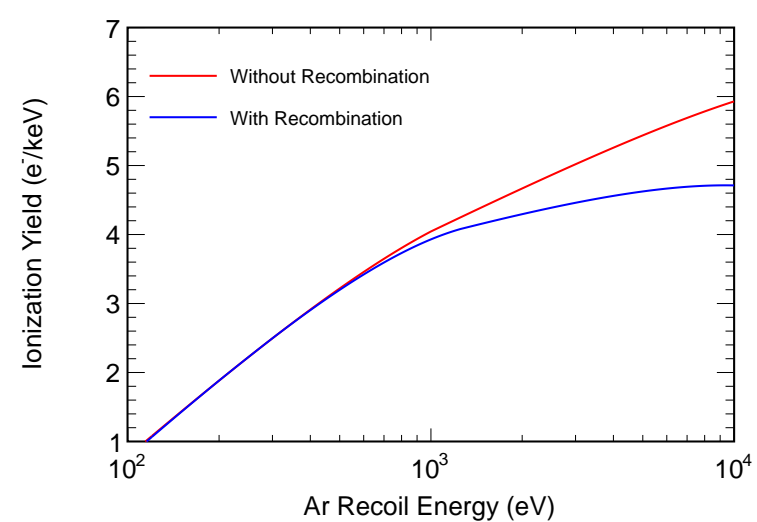

Figure 6: Ionization yield curve for LAr below $10 \mathrm{keV}$ before (red) and after (blue) recombination, as calculated by the Monte Carlo simulation, for $10 \mathrm{eV}$ ionization electrons in an electric field of $1 \mathrm{kV} / \mathrm{cm}$.

\section{Comparison with Experimental Data}

To predict the detectable ionization yield from nu- ${ }^{245}$ clear recoils, the primary ionization yield is combined 246 with the electron separation efficiency. The atomic col- ${ }^{247}$ lision model is used to perform calculations at a given ${ }^{248}$ energy, and the number of ions produced and their respective positions in each trial are used as an input to the electron recombination model. The resulting number of electrons which do not recombine with initial 250 ion cloud is then used to calculate the drift field depen- 251 dent ionization yield. For recoil energies below $\sim 1 \mathrm{keV} 252$ ( $\sim 4$ electrons), electron recombination does not have a 253 significant effect on the ionization yield for drift fields 254 $>0.1 \mathrm{kV} / \mathrm{cm}$. For larger energies, recombination be- 255 comes significant and results in a reduction in the de- 256 tectable electron yield, as shown in Figure 6. The pri- 257 mary scintillation and ionization yields are nearly iden- 258 tical, as both of them are dominated by the neutral Ar-Ar 259 cross sections (shown in Figure 1).

A comparison of the collected number of electrons 261 from the model with those measured for $6.7 \mathrm{keV}$ nuclear 262 recoils in Ref [29] is shown in Figure 7] For currently 263 available data and attained experimental accuracy, the 264 model reproduces both the magnitude and trend of the 265 experimental data points. Moreover, taking into account 266 Penning ionization should bring the 'field dependence ${ }_{267}$ saturation' value to a larger total charge, while the exis- 268 tence of 'near zero energy electrons' in ionization elec- 269 tron energy distribution should increase the 'saturation 270 field' to a higher value. The fraction of 'near zero en- 271 ergy electrons' is unknown due to experimental thresh- 272 olds in Ref [23]. We do not introduce additional fitting 273 parameters to account for the mentioned effects. To im- 274

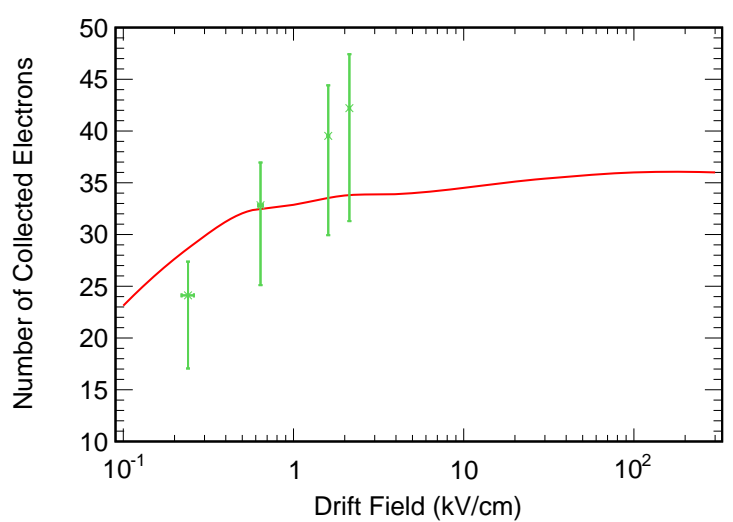

Figure 7: The number of collected electrons shown in Figure 5 in terms of nuclear recoil energy. The green data points are the $6.7 \mathrm{keV}$ nuclear recoil data measured in Ref [29]. The errors on the measured data are too large to distinguish the two ionization yield scenarios shown in Figure 6 (with versus without recombination).

prove the model predicting power, Penning processes cross sections and ionization electrons energy distribution need to be measured in an independent experiment or theoretically estimated.

\section{Ionization Yield from CENNS at a reactor}

Let us consider the CENNS signal resulting from interactions with antineutrinos produced in a nuclear reactor. The antineutrino spectrum emitted from a nuclear reactor depends on the relative contributions of the fissioning nuclides to fission power, since the antineutrino spectrum produced in fission varies with the parent nucleus [30, 31]. In the remainder of this discussion, we assume a 3 gigawatt thermal (GWth) reactor at a 25-m standoff (antineutrino flux is approximately $6 \times 10^{12} \mathrm{~cm}^{-2} \mathrm{~s}^{-1}$ ), and with the same isotopic content as used in Ref. [8].

The net spectrum of detectable electrons is obtained by folding the expected Ar recoil spectrum [8] with the ionization yield and the ionization electron separation efficiency. For our estimate we assumed $E_{\text {elec }}=10 \mathrm{eV}$ and a drift field of $1.0 \mathrm{kV} / \mathrm{cm}$. Most of the recoils $(\sim 70 \%)$ produce no detectable ionization, with the remaining events yielding up to $\sim 5$ electrons. The total expected CENNS rate of both ionizing and non-ionizing events is 56 events per $\mathrm{kg}$ of Ar per day [8]. Figure 8 shows the updated prediction of the CENNS event rates, along with internal backgrounds.

The primary internal background is ${ }^{39} \mathrm{Ar}$, which decays via $\beta$-decay with an end-point energy of $565 \mathrm{keV}$ and a half-life of 269 years. It has been measured 
that the activity of ${ }^{39} \mathrm{Ar}$ is $\sim 1 \mathrm{~Bq} / \mathrm{kg}$ in atmospheric $\operatorname{Ar}[32,33,34]$. Assuming the beta spectrum given in Ref. [35] and a W-value of $23.6 \mathrm{eV}$, we expect the spectral contribution of ${ }^{39} \mathrm{Ar}$ to be flat in the low-energy ("few electron events") region with an amplitude of $\sim 4.1$ events. $\left(e^{-}\right)^{-1} \mathrm{~kg}^{-1} \mathrm{day}^{-1}$, see Figure 8 Efforts have been made to collect Ar from exhaust streams of underground mining facilities, which are depleted in ${ }^{39} \mathrm{Ar}$ by $\sim 150 \times$ that of atmospheric Ar [36]. With an assumed depletion factor of 150 , the background ${ }^{39} \mathrm{Ar}$ rate drops to just 0.027 events. $\left(e^{-}\right)^{-1} \mathrm{~kg}^{-1} \mathrm{day}^{-1}, \sim 100 \times$ lower than the predicted CENNS rates. Due to the low-energy region of interest, there is not enough primary scintillation produced to further reduce the electronic recoil background through pulse shape discrimination.

Both $\mathrm{Ar}$ and Xe dual-phase detectors demonstrate detector-dependent low-energy background at low part of the energy spectra (events where 1-10 electrons were detected), and the number of these events increases with decreasing energy [9, 37]. Single-electron events can be related to trapping of electrons on liquid-gas boundary after "previous" events and subsequent "delayed evapo- ${ }^{32}$ ration". This effect should be stronger for Xe detectors, because LAr has lower electron work function than liq- ${ }^{326}$ uid $\mathrm{Xe}\left(0.21\right.$ and $0.61 \mathrm{eV}$ respectively [38]). Another ${ }^{327}$ mechanism responsible for delayed electrons and pho- ${ }^{328}$ ton production could be associated with trapping of pos- ${ }^{329}$ itive ions in thin physisorbed solid $\mathrm{Ar}(\mathrm{Xe})$ layers on ${ }^{330}$ the surfaces of metal electrodes and their subsequent de- ${ }^{331}$ layed recombination. This question requires additional ${ }^{332}$ detailed investigation and is well beyond the scope of the present paper.

\section{Discussion}

Interpretation of our results is strongly influenced by 339 the sensitivity we are demanding of current and future 340 noble element detectors. We would like to point out that 341 the basic physics behind both charge and light yield at ${ }_{342}$ low energies has never been fully explored with respect ${ }^{343}$ to the relevant atomic and condensed matter physics. 344 This gap in understanding has not prevented obtaining 345 robust results from noble element detectors, but only 346 because previous experiments have limited their anal- 347 ysis to higher energies where the uncertainty of a single ${ }^{348}$ quantum of excitation was overshadowed by larger sys- 349 tematic uncertainties. The state-of-the-art has reached 350 the point, however, where ionization / scintillation de- 351 tectors are sensitive to the single quantum, and indeed ${ }_{352}$ we have already embarked on searches for signals in ${ }_{353}$ this realm. Modeling the behavior of these materials ${ }_{354}$

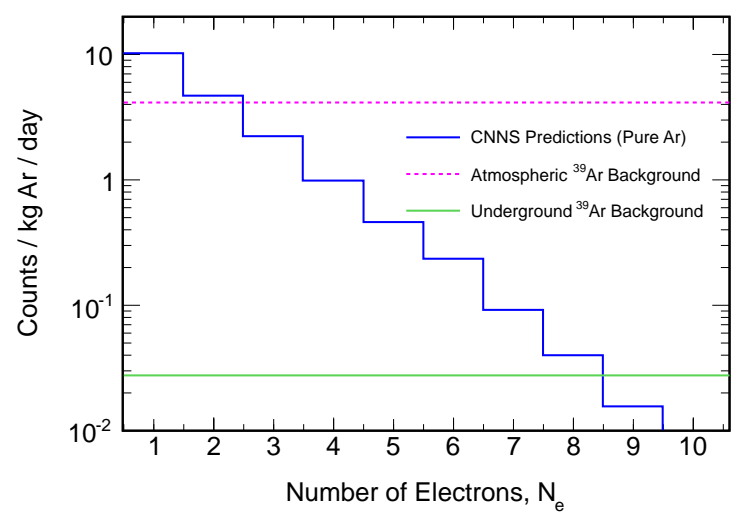

Figure 8: Predicted CENNS event rates from reactor neutrinos for pure LAr (blue), with the backgrounds from ${ }^{39} \mathrm{Ar}$ overlaid. The ${ }^{39} \mathrm{Ar}$ background for atmospheric $\mathrm{Ar}$ (dashed-magenta) is reduced by a factor of $\sim 150$ [36] when underground $\mathrm{Ar}$ is used (solid-green). The event rate assumes a neutrino flux of $6 \times 10^{12} \bar{v} \mathrm{~cm}^{-2} \mathrm{~s}^{-1}$ at a distance of $25 \mathrm{~m}$ from a $3 \mathrm{GW}$ th nuclear reactor core.

down to the single quantum level therefore becomes increasingly important as we push to this absolute limit of detector sensitivity. Understanding the source of backgrounds and parasitic effects is equally important, as their rates and energies compete with the sought-after signals of interest. Just one example of a background to single-quantum signals may come from positive ions trapped in solid noble element layers inside the cryogenic detectors [39].

For a pure Ar detector, the ionization yield for the average expected nuclear recoil energy from CENNS of reactor neutrinos $(250 \mathrm{eV})$ is predicted to be $\sim 2 e^{-} / \mathrm{keV}$. With equal number of excitation and ionization events, there is potential to increase the ionization yield slightly through the utilization of the Penning mechanism in which excited Ar atoms ionize a Penning substance such as Xe $[8,40]$. For $1.6 \%$ Xe doping, the Penning mechanism has a measured conversion efficiency of $\sim 60 \%$ [40]. Due to the short range of recoiled Ar atoms, the Penning ionization of Xe must compete with the Penning ionization of another excited Ar atom or molecule, and thus for low-energy recoils the optimal concentration of Xe may be higher to maximize ionization yield. Even in pure Ar, the short Ar recoil track length - and hence large exciton density-may increase ionization due to bi-excitonic quenching [41]. At present the conversion coefficient is known for $\mathrm{LHe}$ [26] but not for LAr.

The predicted ionization yields suggest that LAr is a plausible candidate for detecting CENNS using reactor antineutrinos. The ${ }^{39} \mathrm{Ar}$ background can be reduced by 
using underground $\mathrm{Ar}$ as the detector medium. Since 405 it is possible that the CENNS signal could be observed ${ }_{406}$ with atmospheric Ar in the reactor on/off scenario, one 407 must consider the "total-cost" comparison of construct- 408 ing a larger detector utilizing atmospheric Ar versus a 409 smaller detector utilizing underground Ar. In addition 410 to the search for CENNS with reactor neutrinos, LAr 411 could also be utilized in the search for other interactions ${ }_{412}$ producing similar low-energy depositions. In any case, ${ }_{413}$ based on our predictions, it does not appear that local 414 electron recombination has a large effect $(\lesssim 5 \%)$ on the ${ }_{415}$ very low energy $(\lesssim 5$ electrons) signals.

The source of low-energy backgrounds in dual-phase ${ }_{417}$ noble element detectors has not been conclusively iden- 418 tified. Multiple experiments have handled this lack of ${ }_{419}$ understanding by applying a lower limit to the anal- 420 ysis threshold (see, e.g., [29], though it is useful to 421 note that all recent searches for weakly interacting mas- 422 sive particles have also applied analysis thresholds). In ${ }_{423}$ particular, Ref. [37] shows that the unattributed back- ${ }^{424}$ ground in a dual-phase argon detector is rising quickly ${ }_{425}$ through $270 \mathrm{eV}$, and this background is in direct competition with the 250-eVee (electron equivalent) CENNS signal from reactor neutrinos. This rapid rise in ionization background at low energy is also seen in dual- ${ }_{427}$ phase xenon detectors [42, 43], leading to the hypoth- ${ }_{428}$ esis that all dual-phase noble element detectors might ${ }_{429}$ share a common effect contributing to the low-energy ${ }_{430}$ background.

\section{Future Work}

While our model represents the first step to develop ${ }_{43}$ an understanding of the intrinsic LAr detector response at low $(<50 \mathrm{keV})$ energies, more work is needed. We have focused solely on the microscopic behavior of $\mathrm{Ar}$ ionization and recombination, but there are detector- ${ }_{437}$ specific effects that must be included in the individual detector response function, such as the effect of trapped ions on detector surfaces, which are beyond the scope of this paper.

The authors note that there may be other approaches to calculating the yield such as a first-principle atomic physics calculation [44]. Regardless of the theoreti- ${ }_{446}^{445}$ cal approach, experiments in the corresponding energy ${ }^{447}$ range must be conducted for validation. Future experi- ${ }^{44}$ ments should not only measure yields and cross sections of relevant interactions such as $\mathrm{Ar}-\mathrm{Ar}, \mathrm{Ar}-\mathrm{Ar}^{+}$, and $\mathrm{Ar}^{*}-{ }_{451}$ Ar, but also measure the rate of Penning ionization as ${ }^{452}$ a function of material composition. Other fundamental ${ }^{453}$ measurements should include the spectra of ionization ${ }_{455}^{454}$ (secondary) electron energies in different collisions, and ${ }_{456}$ the role of multi-body collisions. The authors recommend that the highest priority for calculations and measurements be given to Ar and Xe, given these materials' prevalence in searches for dark matter and CENNS.

Experiments have been recently reported focusing on the measurement of the ionization and scintillation yields in LAr at $<50 \mathrm{keV}$ nuclear recoil energies [29, 45]. The framework presented here should be applicable to other noble elements such as Xe, and a prediction of the ionization yield for CENNS on Xe will be possible when Xe-Xe inelastic cross sections become available.

Other important potential applications for the model exist, related to the operation of dual-phase noble element detectors. The use of a stopped pion neutrino source, such as the spallation neutron source (SNS), would result in greater recoil energies that those produced from reactor antineutrinos [46]. In the search for dark matter, $\mathrm{keV}$-scale energy depositions are anticipated from low-mass weakly interacting massive particles (WIMPs) [47].

\section{Acknowledgements}

This work was performed under the auspices of the U.S. Department of Energy by the Lawrence Livermore National Laboratory under Contract DE-AC5207NA27344. A portion of M. Foxe's research was performed under the Nuclear Forensics Graduate Fellowship Program, which is sponsored by the U.S. Department of Homeland Security, Domestic Nuclear Detection Office and the U.S. Department of Defense, Defense Threat Reduction Agency. PNNL-SA-100229

\section{References}

[1] XENON10 Collaboration, Design and performance of the XENON10 dark matter experiment, Astroparticle Physics 34 (9) (2011) 679-698. doi:10.1016/j.astropartphys.2011. 01.006

[2] XENON100 Collaboration, First dark matter results from the XENON100 experiment, Physical Review Letters 105 (13) (2010) 131302. doi:10.1103/PhysRevLett.105.131302

[3] ZEPLIN-II Collaboration, First limits on WIMP nuclear recoil signals in ZEPLIN-II: a two-phase xenon detector for dark matter detection, Astroparticle Physics 28 (3) (2007) 287-302. doi:10.1016/j.astropartphys.2007.06.002

[4] ZEPLIN Collaboration, The ZEPLIN III dark matter project, New Astronomy Reviews 49 (2-6) (2005) 277-281. doi: $10.1016 / j$.newar.2005.01.018

[5] ArDM Collaboration, The argon dark matter experiment (ArDM), 2008. doi:10.3204/DESY-PROC-2008-02/ regenfus_christian

[6] R. Brunetti et al., WARP liquid argon detector for dark matter survey, New Astronomy Reviews 49 (2-6) (2005) 265-269. doi:10.1016/j.newar.2005.01.017 
[7] D.S. Akerib et al., First Results from the LUX Dark Mat- 522 ter Experiment at the Sanford Underground -Research Facil- 523 ity, Physical Review Letters, 112 (2014) 091303. 10.1103/524 PhysRevLett.112.091303

[8] C. Hagmann, A. Bernstein, Two-phase emission detector for 526 measuring coherent neutrino-nucleus scattering, Nuclear Sci- 527 ence, IEEE Transactions on 51 (5) (2004) 2151 - 2155. doi: 528 10.1109/TNS. 2004.836061

[9] D. Akimov, A. Bondar, A. Burenkov, A. Buzulutskov, Detec- 530 tion of reactor antineutrino coherent scattering off nuclei with a 531 two-phase noble gas detector, Journal of Instrumentation 4 (06) 532 (2009) P06010-P06010. doi:10.1088/1748-0221/4/06/ 533 P06010

[10] T. Takahashi, S. Konno, T. Doke, The average energies, w, re- 535 quired to form an ion pair in liquefied rare gases, Journal of 536 Physics C: Solid State Physics 7 (1974) 230.

[11] T. Doke, A. Hitachi, S. Kubota, A. Nakamoto, T. Takahashi, 538 Estimation of fano factors in liquid argon, krypton, xenon 539 and xenon-doped liquid argon, Nuclear Instruments and Meth- 540 ods 134 (2) (1976) 353-357. doi:10.1016/0029-554X(76) 541 90292-5

[12] W. Walkowiak, Drift velocity of free electrons in liquid ar- 543 gon, Nuclear Instruments and Methods in Physics Research 544 Section A: Accelerators, Spectrometers, Detectors and Asso- 545 ciated Equipment 449 (1-2) (2000) 288-294. doi:10.1016/ 546 S0168-9002(99)01301-7

[13] K. Yoshino, U. Sowada, W. F. Schmidt, Effect of molecular so- 548 lutes on the electron drift velocity in liquid $\mathrm{Ar}, \mathrm{Kr}$, and $\mathrm{Xe}, 549$ Physical Review A 14 (1) (1976) 438-444. doi:10.1103/ 550 PhysRevA.14.438

[14] J. Lindhard, V. Nielsen, M. Scharff, P. Thomsen, Integral equa- 552 tions governing radiation effects (notes on atomic collisions, 553 III).

[15] J. F. Ziegler, Stopping of energetic light ions in elemental matter, 555 Journal of Applied Physics 85 (3) (1999) 1249-1272. doi : doi : 556 10.1063/1.369844

[16] P. Sorensen, C. E. Dahl, Nuclear recoil energy scale in liq- 558 uid xenon with application to the direct detection of dark mat- 559 ter, Physical Review D 83 (6) (2011) 063501. doi:10.1103/ 560 PhysRevD.83.063501

[17] D.-M. Mei, Z.-B. Yin, L. Stonehill, A. Hime, A model of 562 nuclear recoil scintillation efficiency in noble liquids, As- 563 troparticle Physics 30 (1) (2008) 12-17. doi:10.1016/j. 564 astropartphys.2008.06.001

[18] V. Tretyak, Semi-empirical calculation of quenching factors for 565 ions in scintillators, Astroparticle Physics 33 (1) (2010) 40-53. 567 doi:10.1016/j.astropartphys.2009.11.002

[19] M. Szydagis, N. Barry, K. Kazkaz, J. Mock, D. Stolp, 569 M. Sweany, M. Tripathi, S. Uvarov, N. Walsh, M. Woods, 570 NEST: a comprehensive model for scintillation yield in liq- 571 uid xenon, Journal of Instrumentation 6 (10) (2011) P10002- 572 P10002. doi:10.1088/1748-0221/6/10/P10002

[20] M. Foxe, C. Hagmann, I. Jovanovic, A. Bernstein, K. Kazkaz, 574 V. Mozin, S. Pereverzev, S. Sangiorgio, P. Sorensen, Low- 575 energy electron ionization and recombination model for a liq- 576 uid argon detector, Nuclear Instruments and Methods in Physics 577 Research Section A: Accelerators, Spectrometers, Detectors and 578 Associated Equipment 771 (0) (2015) 88 - 92. doi:10.1016/ 579 j.nima.2014.10.055

[21] J. Biersack, L. Haggmark, A monte carlo computer program 581 for the transport of energetic ions in amorphous targets, Nu- 582 clear Instruments and Methods 174 (1-2) (1980) 257-269. doi : 583 10.1016/0029-554X(80)90440-1

[22] A. V. Phelps, Cross sections and swarm coefficients for nitro- 585 gen ions and neutrals in $\mathrm{N}_{2}$ and argon ions and neutrals in $\mathrm{Ar} 586$ for energies from $0.1 \mathrm{eV}$ to $10 \mathrm{keV}$, Journal of Physical and Chemical Reference Data 20 (3) (1991) 557-573. doi:doi: 10.1063/1.555889

[23] G. Gerber, R. Morgenstern, A. Niehaus, Ionization processes in slow collisions of heavy particles II. symmetrical systems of the rare gases $\mathrm{He}, \mathrm{Ne}, \mathrm{Ar}, \mathrm{Kr}$, Journal of Physics B: Atomic and Molecular Physics 6 (3) (1973) 493-510. doi:10.1088/ 0022-3700/6/3/017

[24] M. Wojcik, M. Tachiya, Electron thermalization and electronion recombination in liquid argon, Chemical Physics Letters 379 (1-2) (2003) 20-27. doi:10.1016/j.cplett.2003.08. 006

[25] A. Konovalov, G. Shlyapnikov, The Decay Kinetics of Triplet $\mathrm{He}(23 \mathrm{~S})$ Excitations in Liquid and Solid Helium, Zhurnal Eksperimentalnoi I Teoreticheskoi Fiziki 100 (2) (1991) 521531.

[26] S. Kafanov, A. Parshin, I. Todoshchenko, Structure and dynamics of the $\mathrm{He}_{2}^{*}\left(a^{3} \Sigma_{u}^{+}\right)$molecular complex in condensed phases of helium, Journal of Experimental and Theoretical Physics 91 (5) (2000) 991-999. doi:10.1134/1.1334989

[27] M. H. Cohen, J. Lekner, Theory of hot electrons in gases, liquids, and solids, Physical Review 158 (2) (1967) 305-309. doi:10.1103/PhysRev.158.305

[28] M. Wojcik, M. Tachiya, Electron transport and electron-ion recombination in liquid argon: simulation based on the CohenLekner theory, Chemical Physics Letters 363 (3-4) (2002) 381388. doi:10.1016/S0009-2614(02)01177-6

[29] T. Joshi, S. Sangiorgio, A. Bernstein, M. Foxe, C. Hagmann, I. Jovanovic, K. Kazkaz, V. Mozin, E. Norman, S. Pereverzev, F. Rebassoo, P. Sorensen, First measurement of the ionization yield of nuclear recoils in liquid argon, Physical Review Letters 112 (17) (2014) 171303. doi:10.1103/PhysRevLett.112. 171303

[30] P. Vogel, J. Engel, Neutrino electromagnetic form factors, Physical Review D 39 (11) (1989) 3378-3383. doi:10.1103/ PhysRevD.39.3378

[31] P. Huber, T. Schwetz, Precision spectroscopy with reactor antineutrinos, Physical Review D 70 (5) (2004) 053011. doi: 10.1103/PhysRevD.70.053011

[32] H. Loosli, A dating method with ${ }^{39} \mathrm{Ar}$, Earth and Planetary Science Letters 63 (1) (1983) 51-62. doi:10.1016/ 0012-821X (83)90021-3

[33] W. Kutschera, M. Paul, I. Ahmad, T. Antaya, P. Billquist, B. Glagola, R. Harkewicz, M. Hellstrom, D. Morrissey, R. Pardo, K. Rehm, B. Sherrill, M. Steiner, Long-lived noble gas radionuclides, Nuclear Instruments and Methods in Physics Research Section B: Beam Interactions with Materials and Atoms 92 (1-4) (1994) 241-248. doi:10.1016/0168-583X(94) 96013-5

[34] P. Benetti, F. Calaprice, E. Calligarich, M. Cambiaghi, F. Carbonara, F. Cavanna, A. Cocco, F. Di Pompeo, N. Ferrari, G. Fiorillo, C. Galbiati, L. Grandi, G. Mangano, C. Montanari, L. Pandola, A. Rappoldi, G. Raselli, M. Roncadelli, M. Rossella, C. Rubbia, R. Santorelli, A. Szelc, C. Vignoli, Y. Zhao, Measurement of the specific activity of ${ }^{39} \mathrm{Ar}$ in natural argon, Nuclear Instruments and Methods in Physics Research Section A: Accelerators, Spectrometers, Detectors and Associated Equipment 574 (1) (2007) 83-88. doi:10.1016/j.nima.2007.01. 106

[35] M. Stabin, L. da Luz, Decay data for internal and external dose assessment, Health Physics 83 (4) (2002) 471-475. doi:10. 1097/00004032-200210000-00004

[36] J. Xu, F. Calaprice, C. Galbiati, A. Goretti, G. Guray, T. Hohman, D. Holtz, A. Ianni, M. Laubenstein, B. Loer, C. Love, C. J. Martoff, D. Montanari, S. Mukhopadhyay, A. Nel- 
son, S. D. Rountree, R. B. Vogelaar, A. Wright, A study of the residual 39Ar content in argon from underground sources arXiv:1204.6011 [hep-ex, physics:nucl-ex, physics:physics]. URL http://arxiv.org/abs/1204.6011

[37] S. Sangiorgio, T. Joshi, A. Bernstein, J. Coleman, M. Foxe, C. Hagmann, I. Jovanovic, K. Kazkaz, K. Mavrokoridis, V. Mozin, S. Pereverzev, P. Sorensen, First demonstration of a sub-kev electron recoil energy threshold in a liquid argon ionization chamber, Nuclear Instruments and Methods in Physics Research Section A: Accelerators, Spectrometers, Detectors and Associated Equipment 728 (0) (2013) 69 - 72. doi : 10.1016/ j.nima.2013.06.061

[38] E. Aprile, A. E. Bolotnikov, A. Bolozdynya, T. Doke, Hot electron emission, in: Noble Gas Detectors, WILEY-VCH Verlag GmbH \& Co. KGaA, Weinheim, 2006, pp. 62-64.

[39] B. Rebel et al., High voltage in noble liquids for high energy physics, Journal of Instrumentation, 9 (2014) T08004. doi: 10.1088/1748-0221/9/08/T08004

[40] S. Kubota, A. Nakamoto, T. Takahashi, S. Konno, T. Hamada, M. Miyajima, A. Hitachi, E. Shibamura, T. Doke, Evidence of the existence of exciton states in liquid argon and excitonenhanced ionization from xenon doping, Physical Review B 13 (4) (1976) 1649-1653. doi:10.1103/PhysRevB.13. 1649

[41] A. Hitachi, T. Doke, A. Mozumder, Luminescence quenching in liquid argon under charged-particle impact: Relative scintillation yield at different linear energy transfers, Physical Review B 1 46 (18) (1992) 11463-11470. doi:10.1103/PhysRevB.46. 11463

[42] P. Sorensen et al., Lowering the low-energy threshold of xenon detectors, Proceedings of Science, IDM2010 017 (2010).

[43] D.Yu. Akimov et al., Experimental study of ionization yield of liquid xenon for electron recoils in the energy range $2.8-80 \mathrm{keV}$, Journal of Instrumentation, 9 (2014) P11014. doi:10.1088/ 1748-0221/9/11/P11014

[44] Personal communications with M. Szydagis.

[45] T. Alexander, H. O. Back, H. Cao, A. G. Cocco, F. DeJongh, G. Fiorillo, C. Galbiati, C. Ghag, L. Grandi, C. Kendziora, W. H. Lippincott, B. Loer, C. Love, L. Manenti, C. J. Martoff, Y. Meng, D. Montanari, P. Mosteiro, D. Olvitt, S. Pordes, H. Qian, B. Rossi, R. Saldanha, W. Tan, J. Tatarowicz, S. Walker, H. Wang, A. W. Watson, S. Westerdale, J. Yoo, Observation of the dependence of scintillation from nuclear recoils in liquid argon on drift field Physical Review D 88 (9), arXiv:1306.5675 [hep-ex, physics:physics]. doi:10.1103/ PhysRevD.88.092006 URL http://arxiv.org/abs/1306.5675

[46] K. Scholberg, Prospects for measuring coherent neutrinonucleus elastic scattering at a stopped-pion neutrino source Physical Review D 73 (3) (2006) 033005. doi:10.1103/ PhysRevD.73.033005

[47] P. Fox, G. Jung, P. Sorensen, N. Weiner, Dark matter in light of the LUX results, Physical Review D 89 (2014) 103526. doi: 10.1103/PhysRevD.89.103526 